\title{
Effect of long-term low-dose folic acid supplementation on degree of total homocysteine-lowering: major effect modifiers
}

\author{
Binyan Wang ${ }^{1,2}$, Hongxu Wu ${ }^{1}$, Youbao $\mathrm{Li}^{2}$, Qianyun Ban ${ }^{1}$, Xiao Huang ${ }^{3}$, Lulu Chen ${ }^{1}$, Jianping $\mathrm{Li}^{4}$, \\ Yan Zhang ${ }^{4}$, Yimin Cui ${ }^{5}$, Mingli He ${ }^{6}$, Genfu Tang ${ }^{1}$, Delu Yin ${ }^{7}$, Junnong Li ${ }^{8}$, Yong Huo ${ }^{3}$, Xianhui Qin ${ }^{1,2_{*}}$ \\ and Xiping $\mathrm{Xu}^{1,2 *}$ \\ ${ }^{1}$ Institute for Biomedicine, Anhui Medical University, Hefei 230032, People's Republic of China \\ ${ }^{2}$ National Clinical Research Center for Kidney Disease, State Key Laboratory for Organ Failure Research, Key Laboratory of \\ Organ Failure Research (Ministry of Education), Renal Division, Nanfang Hospital, Southern Medical University, Guangzhou \\ 510515, People's Republic of China \\ ${ }^{3}$ Department of Cardiology, Second Affiliated Hospital, Nanchang University, Nanchang 330006, People's Republic of China \\ ${ }^{4}$ Department of Cardiology, Peking University First Hospital, Beijing 100034, People's Republic of China \\ ${ }^{5}$ Department of Pharmacy, Peking University First Hospital, Beijing 100034, People's Republic of China \\ ${ }^{6}$ Department of Neurology, First People's Hospital, Lianyungang 222061, People's Republic of China \\ ${ }^{7}$ Department of Cardiology, First People's Hospital, Lianyungang 222061, People's Republic of China \\ ${ }^{8}$ Department of Cardiology, Weinan Center Hospital, Weinan 714000, People's Republic of China
}

(Submitted 5 February 2018 - Final revision received 3 June 2018 - Accepted 6 August 2018)

\section{Abstract}

We sought to examine the potential modifiers in the association between long-term low-dose folic acid supplementation and the reduction of serum total homocysteine (tHcy) among hypertensive patients, using data from the China Stroke Primary Prevention Trial (CSPPT). This analysis included 16867 participants who had complete data on tHcy measurements at both the baseline and exit visit. After a median treatment period of 4.5 years, folic acid treatment significantly reduced the tHcy levels by $1.6 \mu \mathrm{mol} / 1$ (95\% CI 1.4, 1.8). More importantly, after adjustment for baseline tHcy and other important covariates, a greater degree of tHcy reduction was observed in certain subgroups: males, the methylenetetrahydrofolate reductase (MTHFR) 677TT genotype, higher baseline tHcy levels $(\geq 12.5$ (median) $v .<12.5 \mu \mathrm{mol} / \mathrm{l})$, lower folate levels $\left(<8.0\right.$ (median) $v$. $\geq 8.0 \mathrm{ng} / \mathrm{ml}$ ), estimated glomerular filtration rate (eGFR) $<60 \mathrm{ml} / \mathrm{min}$ per $1.73 \mathrm{~m}^{2}(v .60-<90$ and $\geq 90 \mathrm{ml} / \mathrm{min}$ per $1.73 \mathrm{~m}^{2}$ ), ever smokers and concomitant use of diuretics ( $P$ for all interactions $<0.05$ ). The degree of tHcy reduction associated with long-term folic acid supplementation can be significantly affected by sex, MTHFR C677T genotypes, baseline folate, tHcy, eGFR levels and smoking status.

Key words: Folic acid: Total homocysteine: Effect modifiers: Hypertension

Increased plasma total homocysteine (tHcy) has been reported to be associated with a variety of clinical outcomes, including hypertension, diabetes, chronic kidney diseases, CVD, cancer, psychiatric disorders and cognitive impairment ${ }^{(1-3)}$. It has been reported that hypertension and raised tHcy can synergistically increase the risk of stroke ${ }^{(4,5)}$. Furthermore, a significant positive dose-response association has been found between degree of tHcy reduction and systolic blood pressure (SBP) reduction $^{(6)}$, and decrease in $\mathrm{CVD}^{(7)}$ or carotid intima-media thickness $^{(8)}$. Consistently, a recent study found that the lowering of tHcy was significantly associated with a reduction in first stroke risk, and may possibly serve as a useful indicator for the use of folic acid therapy in the prevention of $\operatorname{stroke}^{(9)}$.

In previous randomised trials with long-term folic acid therapy, the percentage of tHcy reduction between groups ranged from 10 to $38 \%^{(10-12)}$. Many of these previous trials were conducted in participants with CVD, and a high dose of folic acid (median: $2 \mathrm{mg}$ ) was used, often combined with vitamin $\mathrm{B}_{12}$ and/or $\mathrm{B}_{6}{ }^{(10-12)}$. In fact, a previous meta-analysis of randomised trials suggested that daily doses of $0.8 \mathrm{mg}$ of folic acid had achieved the maximum reduction in serum tHcy levels ${ }^{(13)}$. Little further tHcy reduction is achieved by increasing the dose of

Abbreviations: CCB, calcium channel blockers; CSPPT, China Stroke Primary Prevention Trial; eGFR, estimated glomerular filtration rate; MTHFR, methylenetetrahydrofolate reductase; SBP, systolic blood pressure; tHcy, total homocysteine.

* Corresponding authors: X. Qin, fax +86 551 5161211, email pharmaqin@126.com; X. Xu, fax +86 551 5161211, email xipingxu126@126.com 
folic acid above $0.8 \mathrm{mg} / \mathrm{d}^{(13)}$. Furthermore, two recent metaanalyses of randomised trials found that folic acid therapy significantly reduced the risk of stroke by more than $20 \%$ among trials with a daily folic acid dosage of $0.8 \mathrm{mg}$ or lower. Excessive doses of folic acid therapy did not lead to an additional beneficial effect ${ }^{(12)}$. More importantly, there has been a lack of randomised clinical trials that have comprehensively assessed the modifiers affecting the variation of tHcy reduction associated with long-term folic acid supplementation. The aim of the present study was to fill in these important gaps in knowledge regarding folic acid supplementation and the reduction of tHcy. A better understanding of the modifiable risk factors would be helpful for clinical and public health decision-making, for it would lead to early identification and effective risk reduction, and result in an individualised folic acid treatment regimen with the best benefit-risk ratio.

The main analyses of the China Stroke Primary Prevention Trial (CSPPT), which have been published previously, showed that folic acid therapy can reduce the risk of first stroke by $21 \%$ among hypertensive patients ${ }^{(14)}$. The present post hoc analysis of the CSPPT aimed to examine the potential modifiers in the association between long-term low-dose folic acid supplementation and the reduction of tHcy among hypertensive patients without major CVD, using data from the CSPPT.

\section{Methods}

\section{Study participants}

The methods and primary results of the CSPPT have been reported elsewhere ${ }^{(14)}$. Briefly, the CSPPT was a multi-community, randomised, double-blind, controlled trial conducted from 19 May 2008 to 24 August 2013 in thirty-two communities in Jiangsu and Anhui provinces in China. Eligible participants were men and women aged $45-75$ years old who had hypertension, defined as seated resting SBP $\geq 140 \mathrm{mmHg}$ or diastolic blood pressure (DBP) $\geq 90 \mathrm{mmHg}$ at both the screening and recruitment visit, or were on antihypertensive medication. The major exclusion criteria included history of physician-diagnosed stroke, myocardial infarction (MI), heart failure, post-coronary revascularisation or congenital heart disease.

The present study was approved by the ethics committee of the Institute of Biomedicine, Anhui Medical University, Hefei, China (FWA assurance number FWA00001263), and was registered with ClinicalTrials.gov, NCT00794885. All participants gave written informed consent before data collection.

\section{Intervention and follow-up}

Eligible participants, stratified by methylenetetrahydrofolate reductase (MTHFR) C677T genotypes (CC, CT or TT), were randomly assigned, in a 1:1 ratio, to one of two treatment groups: a daily oral dose of one tablet containing $10 \mathrm{mg}$ of enalapril and $0.8 \mathrm{mg}$ of folic acid (single pill combination, the enalapril-folic acid group) or a daily oral dose of one tablet containing $10 \mathrm{mg}$ of enalapril only (the enalapril group). All study investigators and participants were blinded to the randomisation procedure and the treatment assignments. During the trial period, concomitant use of other antihypertensive drugs (mainly Ca channel blockers (CCB) or diuretics) was allowed, but not B-vitamins.

Participants were followed up every 3 months. At each visit, participant blood pressure and pulse were measured, the number of pills taken between visits was counted and the use of concomitant medications and any adverse events were recorded.

\section{Laboratory assays}

Laboratory tests were performed at the core laboratory of the National Clinical Research Center for Kidney Disease (Nanfang Hospital, Guangzhou, China). Serum tHcy, fasting lipids and glucose were measured using automatic clinical analysers (Beckman Coulter). tHcy measurement was calibrated to the National Institute of Standards and Technology standard reference material SRM1955. MTHFR C677T polymorphisms were detected on an ABI PRISM ${ }^{\circledR} 7900$ HT sequence detection system (Life Technologies) using the TaqMan assay. Serum folate and $\mathrm{B}_{12}$ at both the baseline and the exit visit were measured by a commercial laboratory using a chemiluminescent immunoassay (New Industrial)

Estimated glomerular filtration rate (eGFR) was calculated using the Chronic Kidney Disease Epidemiology Collaboration equation.

\section{Major definitions}

Hyperuricaemia was defined as a uric acid concentration $\geq 417 \mu \mathrm{mol} / \mathrm{l}(7 \mathrm{mg} / \mathrm{dl})$ in men or $\geq 357 \mu \mathrm{mol} / \mathrm{l}(6 \mathrm{mg} / \mathrm{dl})$ in women. A current smoker was defined as smoking at least one cigarette per $\mathrm{d}$ or smoking more than eighteen packs in the last year. Those who had smoked more than eighteen packs but did not smoke in the last year were defined as former smokers. We combined former smokers and current smokers into an eversmoker group. Regular concomitant medication use was defined as taking the drug of interest for 180 or more cumulative days ${ }^{(15)}$.

We defined metabolic vitamin $\mathrm{B}_{12}$ deficiency ${ }^{(16)}$ as a serum vitamin $B_{12}$ level of $<400 \mathrm{pmol} / 1(500 \mathrm{pg} / \mathrm{ml})$ with normal renal function $\left(\geq 60 \mathrm{ml} / \mathrm{min}\right.$ per $\left.1.73 \mathrm{~m}^{2}\right)$, a thcy level of $\geq 14 \mu \mathrm{mol} / 1$ and a folate level of $10.4 \mathrm{ng} / \mathrm{ml}$ (75\% percentile) or more.

\section{Statistical analysis}

Means (standard deviations) and proportions were calculated for population characteristics by treatment group. Differences in population characteristics were compared using two-sample $t$ tests, signed rank tests or $\chi^{2}$ tests, accordingly.

The treatment effect, expressed as the difference in tHcy level at the exit visit minus that at baseline, was estimated using generalised linear regression models with adjustment for age, sex, study centre, use of angiotensin-converting enzyme inhibitors (ACEI), MTHFR C677T genotypes, smoking status, BMI, tHcy, fasting glucose, total cholesterol, eGFR, folate, vitamin $\mathrm{B}_{12}$, uric acid and SBP at baseline, as well as time-averaged SBP, concomitant use of CCB and concomitant use of diuretics during the trial period. In additional exploratory analyses, possible 
modifications of the treatment effects on the primary outcome were assessed for the variables, including age $(<60 v$ v $\geq 60$ years), sex, MTHFR C677T genotypes, smoking (never $v$. ever smoking), hyperuricaemia (yes $v$. no), folate $(<8.0$ (median) $v$. $\geq 8.0 \mathrm{ng} / \mathrm{ml}$ ), tHcy $(<12.5$ (median), $\geq 12.5 \mu \mathrm{mol} / \mathrm{l})$, vitamin $\mathrm{B}_{12}$ $(<380 \quad$ (median) $v . \quad \geq 380 \mathrm{pg} / \mathrm{ml}), \quad$ eGFR $\quad(<60, \quad 60-<90$ and $\geq 90 \mathrm{ml} / \mathrm{min}$ per $\left.1.73 \mathrm{~m}^{2}\right)$, SBP at baseline $(<160 v$. $\geq 160 \mathrm{mmHg})$, as well as time-averaged $\operatorname{SBP}(<140 v$. $\geq 140 \mathrm{mmHg}$ ) and concomitant use of diuretics (yes $v$. no) during the trial period.

A two-tailed $P<0.05$ was considered statistically significant in all analyses. R software, version 3.3.1 (http://www.R-project. org/), was used for all statistical analyses.

\section{Results}

\section{Study participants and baseline characteristics}

As illustrated in the flow chart (online Supplementary Fig. S1), a total of 20702 participants were enrolled and randomised in the CSPPT. Of those, 10348 participants were assigned to the enalapril-folic acid group and 10354 participants to the enalapril group. We excluded participants who had missing data on tHcy measurements at the baseline ( $n$ 278) or exit visit ( $n$ 3557), leaving 16867 ( $n 8418$ in the enalapril-folic acid group and $n 8449$ in the enalapril group) participants in the final analysis. Participants not included in the analyses did not differ in baseline characteristics from those included in the analyses (online Supplementary Table S1).

All baseline characteristics were comparable between the enalapril and enalapril-folic acid groups (Table 1). There were 1572 (9.3\%) participants undergoing treatment with ACEI at baseline.

\section{Blood pressure at baseline and during the treatment period}

Mean SBP and DBP were highly comparable between the two groups at baseline and over the course of the trial (online Supplementary Tables S2 and S3).

\section{Concomitant medications}

The most common concomitant medications were CCB and diuretics, which were used by 82 and $53 \%$ of the participants, respectively. There were no significant differences in concomitant medication use during the trial between the two treatment groups. The use of lipid-lowering, glucose-lowering and anti-platelet medications were very rare, all at a frequency of $<2 \%$ (online Supplementary Table S4).

\section{Effect of folic acid therapy on change in total homocysteine levels}

Mean baseline tHcy levels were comparable between the two groups (enalapril group, 14.5 (sD 8.5) $\mu \mathrm{mol} / \mathrm{l}$; enalapril-folic acid group, 14.4 (SD 8.1) $\mu \mathrm{mol} / \mathrm{l})$. After a median treatment period of 4.5 years, mean tHcy was 14.6 (SD 8.0) $\mu \mathrm{mol} / \mathrm{l}$ in the enalapril group compared with 12.9 (sD 6.3) $\mu \mathrm{mol} / 1$ in the enalapril-folic acid group (group difference: $-1.6 \mu \mathrm{mol} / 1 ; 95 \%$ CI $-1 \cdot 8,-1.4 \mu \mathrm{mol} / \mathrm{l})$.

As expected, a greater group difference was found in participants with higher baseline tHcy levels ( $\geq 12 \cdot 5$ (median): $-2 \cdot 4$; $95 \%$ CI $-2.9,-2 \cdot 0 ; \quad v .<12.5 \mu \mathrm{mol} / 1:-0.7 ; 95 \%$ CI -0.9 , $-0.6 \mu \mathrm{mol} / \mathrm{l}$ ) (Table 2).

Furthermore, although the group differences were all significant in participants with lower $(<12.5 \mu \mathrm{mol} / \mathrm{l})$ or higher $(\geq 12.5 \mu \mathrm{mol} / \mathrm{l})$ baseline tHcy levels, tHcy increased in those with baseline tHcy $<12.5 \mu \mathrm{mol} / \mathrm{l}$, whereas it decreased in those with baseline tHcy $\geq 12.5 \mu \mathrm{mol} / \mathrm{l}$ in both treatment groups.

\section{Stratified analyses by potential effect modifiers}

Stratified analyses were performed to assess the treatment effect on the primary outcome in various subgroups (Fig. 1). After adjustment for baseline tHcy and other important covariates (age, sex, study centre, use of ACEI, MTHFR C677T genotypes, smoking status, BMI, fasting glucose, total cholesterol, eGFR, folate, vitamin $\mathrm{B}_{12}$, uric acid and $\mathrm{SBP}$ at baseline, as well as time-averaged SBP, concomitant use of CCB and concomitant use of diuretics during the trial period), a greater degree of tHcy reduction was observed in certain subgroups: males, the MTHFR 677TT genotype, higher baseline tHcy levels, lower folate levels, eGFR $<60 \mathrm{ml} / \mathrm{min}$ per $1.73 \mathrm{~m}^{2}$, concomitant use of diuretics and ever smokers ( $P$ for all interactions <0.05). However, SBP either at baseline or over the treatment period, baseline use of ACEI and concomitant use of CCB had no significant effect on the efficacy of folic acid therapy in lowering tHcy levels (Fig. 1 and online Supplementary Table S5).

When split into two groups according to baseline tHcy, similar trends for the same modifiers were found in participants with higher $(\geq 12.5 \mu \mathrm{mol} / \mathrm{l})$ (Fig. 2$)$ or lower $(<12.5 \mu \mathrm{mol} / \mathrm{l})$ (Fig. 3) tHcy levels.

The prevalence of metabolic $\mathrm{B}_{12}$ deficiency was about $4.0 \%$. The tHcy-lowering effect of folic acid therapy was attenuated and became insignificant in participants with metabolic $\mathrm{B}_{12}$ deficiency (online Supplementary Table S6).

\section{Discussion}

Our present analysis is the first study to comprehensively evaluate the effect modifiers of long-term daily $0 \cdot 8$-mg folic acid supplementation in lowering tHcy levels among adults with hypertension without stroke and MI. As expected, long-term daily $0 \cdot 8$-mg folic acid supplementation significantly lowered tHcy levels in this population. More importantly, male, MTHFR 677TT genotype, higher baseline tHcy levels, lower folate levels, eGFR $<60 \mathrm{ml} / \mathrm{min}$ per $1.73 \mathrm{~m}^{2}$, ever smokers and concomitant use of diuretics were major modifiers for the efficacy of folic acid supplementation in lowering tHcy levels. All these factors should be taken into account in the identification of participants who may be most likely to benefit from tHcylowering therapy.

Serum tHcy concentration is determined by genetic and a series of environmental factors. MTHFR is a critical enzyme for folate and tHcy metabolism. MTHFR converts 5,10- 
Table 1. Baseline characteristics of the China Stroke Primary Prevention Trial study participants*

(Numbers and percentages; mean values and standard deviations; medians and 25th-75th percentiles)

\begin{tabular}{|c|c|c|c|c|c|c|}
\hline \multirow[b]{2}{*}{ Variables } & \multicolumn{2}{|c|}{ Total } & \multicolumn{2}{|c|}{ Enalapril } & \multicolumn{2}{|c|}{ Enalapril-folic acid } \\
\hline & $n$ & $\%$ & $n$ & $\%$ & $n$ & $\%$ \\
\hline$n$ & 16867 & & 8449 & & 8418 & \\
\hline Male & 6794 & $40 \cdot 3$ & 3408 & $40 \cdot 3$ & 3386 & $40 \cdot 2$ \\
\hline \multicolumn{7}{|l|}{ Age (years) } \\
\hline Mean & \multicolumn{2}{|c|}{$60 \cdot 0$} & \multicolumn{2}{|c|}{$60 \cdot 0$} & \multicolumn{2}{|c|}{$60 \cdot 1$} \\
\hline SD & \multicolumn{2}{|c|}{$7 \cdot 4$} & \multicolumn{2}{|c|}{$7 \cdot 4$} & \multicolumn{2}{|c|}{$7 \cdot 4$} \\
\hline \multicolumn{7}{|l|}{ BMI $\left(\mathrm{kg} / \mathrm{m}^{2}\right)$} \\
\hline Mean & \multicolumn{2}{|c|}{$25 \cdot 1$} & \multicolumn{2}{|c|}{$25 \cdot 0$} & \multicolumn{2}{|c|}{$25 \cdot 1$} \\
\hline SD & \multicolumn{2}{|c|}{3.7} & \multicolumn{2}{|c|}{3.7} & \multicolumn{2}{|c|}{3.7} \\
\hline Systolic blood pressure (mmHg) & & & & & & \\
\hline Mean & & & & & & \\
\hline SD & & & & & & \\
\hline Diastolic blood pressure $(\mathrm{mmHg})$ & & & & & & \\
\hline Mean & & & & & & \\
\hline SD & & & & & & \\
\hline MTHFR C677T polymorphisms & & & & & & \\
\hline $\mathrm{CC}$ & 4560 & $27 \cdot 0$ & 2275 & $26 \cdot 9$ & 2285 & $27 \cdot 1$ \\
\hline $\mathrm{CT}$ & 8279 & $49 \cdot 1$ & 4148 & $49 \cdot 1$ & 4131 & $49 \cdot 1$ \\
\hline TT & 4028 & 23.9 & 2026 & $24 \cdot 0$ & 2002 & $23 \cdot 8$ \\
\hline Smoking status & & & & & & \\
\hline Never & 11725 & 69.5 & 5875 & 69.5 & 5850 & $69 \cdot 5$ \\
\hline Former & 1273 & $7 \cdot 6$ & 655 & $7 \cdot 8$ & 618 & $7 \cdot 3$ \\
\hline Current & 3862 & 22.9 & 1918 & $22 \cdot 7$ & 1944 & $23 \cdot 1$ \\
\hline Laboratory results (mmol/l) & & & & & & \\
\hline Total cholesterol & & & & & & \\
\hline Mean & & & & & & \\
\hline SD & & & & & & \\
\hline TAG & & & & & & \\
\hline Mean & & & & & & \\
\hline SD & & & & & & \\
\hline HDL-cholesterol & & & & & & \\
\hline Mean & & & & & & \\
\hline SD & & & & & & \\
\hline Fasting glucose & & & & & & \\
\hline Mean & & & & & & \\
\hline SD & & & & & & \\
\hline Vitamin $\mathrm{B}_{12}(\mathrm{pg} / \mathrm{ml})$ & & & & & & \\
\hline Median & & & & & & \\
\hline 25th-75th percentile & & & & & & \\
\hline Folate (ng/ml) & & & & & & \\
\hline Median & & & & & & \\
\hline 25th-75th percentile & & & & & & \\
\hline eGFR $\left(\mathrm{ml} / \mathrm{min}\right.$ per $\left.1.73 \mathrm{~m}^{2}\right)$ & & & & & & \\
\hline Mean & & & & & & \\
\hline SD & & & & & & \\
\hline Medication use & & & & & & \\
\hline Antihypertensive drugs & 7903 & $46 \cdot 9$ & 3980 & $47 \cdot 1$ & 3923 & $46 \cdot 6$ \\
\hline ACE inhibitors & 1572 & $9 \cdot 3$ & 783 & $9 \cdot 3$ & 789 & $9 \cdot 4$ \\
\hline Angiotensin II type I receptor blockers & 15 & 0.1 & 7 & $0 \cdot 1$ & 8 & $0 \cdot 1$ \\
\hline Ca channel blockers & 1674 & 9.9 & 830 & $9 \cdot 8$ & 844 & $10 \cdot 0$ \\
\hline Diuretics & 362 & $2 \cdot 1$ & 184 & $2 \cdot 2$ & 178 & $2 \cdot 1$ \\
\hline$\beta$-Blockers & 147 & 0.9 & 78 & 0.9 & 69 & 0.8 \\
\hline Lipid-lowering drugs & 142 & 0.8 & 73 & 0.9 & 69 & 0.8 \\
\hline Glucose-lowering drugs & 261 & 1.5 & 117 & 1.4 & 144 & 1.7 \\
\hline
\end{tabular}

MTHFR, methylenetetrahydrofolate reductase; eGFR, estimated glomerular filtration rate; ACE, angiotensin-converting enzyme.

* For continuous variables, values are presented as means and standard deviations.

methylenetetrahydrofolate into 5-methyltetrahydrofolate, which is required for the remethylation of tHcy. The polymorphism of MTHFR $677 \mathrm{C} \rightarrow \mathrm{T}$ leads to a reduction in enzyme activity, resulting in increased concentrations of tHcy and lower levels of serum folate ${ }^{(2)}$. Serum tHcy increases with age, and men usually have higher levels than women with the same age. The sexrelated tHcy difference may be partly explained by the effect of oestrogen in women ${ }^{(17)}$. Furthermore, patients with chronic kidney diseases have lower levels of folate owing to inadequate intake, increased loss or demand, and usually have higher tHcy levels. At the same time, tobacco smoke exposure is associated with higher tHcy levels owing to decreased folate intake ${ }^{(18,19)}$ and increased folate turnover ${ }^{(20,21)}$. Accordingly, our study also suggests that participants with the MTHFR 677 TT genotype, 
Table 2. Mean total homocysteine levels $(\mu \mathrm{mol} / \mathrm{l})$ at baseline and exit visit by treatment group (Mean values and standard deviations; mean values and $95 \%$ confidence intervals)

\begin{tabular}{|c|c|c|c|c|c|}
\hline & \multicolumn{2}{|c|}{ Enalapril } & \multicolumn{2}{|c|}{ Enalapril-folic acid } & \multirow[b]{2}{*}{$P$} \\
\hline & Mean & SD & Mean & SD & \\
\hline \multicolumn{6}{|l|}{ All participants } \\
\hline$n$ & \multicolumn{2}{|c|}{8449} & \multicolumn{2}{|c|}{8418} & \\
\hline At baseline & 14.5 & 8.5 & $14 \cdot 4$ & $8 \cdot 1$ & 0.633 \\
\hline At exit & 14.6 & 8.0 & $12 \cdot 9$ & $6 \cdot 3$ & $<0.001$ \\
\hline Change $\dagger$ & 0.1 & $7 \cdot 1$ & $-1.5^{\star}$ & $7 \cdot 7$ & $<0.001$ \\
\hline Change by group & & & -1.6 & & \\
\hline $95 \% \mathrm{Cl}$ & & \multicolumn{4}{|c|}{$-1 \cdot 8,-1.4$} \\
\hline \multirow{2}{*}{\multicolumn{6}{|c|}{$\begin{array}{l}\text { Baseline total homocysteine } \\
<12.5 \mathrm{umol} / \mathrm{l} \text { (median) }\end{array}$}} \\
\hline & & & & & \\
\hline$n$ & \multicolumn{2}{|c|}{4260} & \multicolumn{2}{|c|}{4189} & \\
\hline At baseline & $10 \cdot 2$ & 1.5 & $10 \cdot 2$ & 1.5 & 0.699 \\
\hline At exit & 11.6 & 3.0 & $10 \cdot 8$ & 3.5 & $<0.001$ \\
\hline Change† & $1 \cdot 3^{*}$ & 2.8 & $0.6^{*}$ & $3 \cdot 4$ & $<0.001$ \\
\hline Change by group & & & -0.7 & & \\
\hline $95 \% \mathrm{Cl}$ & & \multirow{2}{*}{\multicolumn{4}{|c|}{$-0.9,-0.6$}} \\
\hline$\geq 12.5 \mu \mathrm{mol} / \mathrm{l}$ & & & & & \\
\hline$n$ & \multicolumn{2}{|c|}{4189} & \multicolumn{2}{|c|}{4229} & \\
\hline At baseline & $18 \cdot 7$ & $10 \cdot 4$ & $18 \cdot 5$ & 9.7 & 0.314 \\
\hline At exit & $17 \cdot 6$ & $10 \cdot 1$ & $15 \cdot 0$ & $7 \cdot 7$ & $<0.001$ \\
\hline Change $†$ & $-1 \cdot 1^{*}$ & 9.5 & $-3 \cdot 5^{\star}$ & 9.8 & $<0.001$ \\
\hline Change by group & \multirow{2}{*}{\multicolumn{5}{|c|}{ 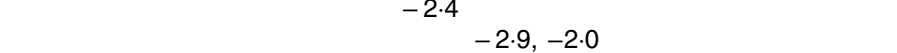 }} \\
\hline $95 \% \mathrm{Cl}$ & & & & & \\
\hline
\end{tabular}

* Significantly different from baseline $(P<0.05)$.

$\dagger$ Change $=$ total homocysteine level at exit - total homocysteine level at baseline.

lower folate or eGFR levels and those who are males and ever smokers seemed to have higher tHcy levels at baseline (Fig. 1). However, it is still unknown whether these factors modify the effect of long-term folic acid supplementation in reducing tHcy.

Consistent with previous studies ${ }^{(13,22)}$, baseline tHcy concentration was the major determinant of tHcy-lowering after folic acid treatment in the present study. A greater tHcy reduction was found in participants with higher baseline tHcy levels. More importantly, although the group difference was also significant among participants with lower baseline tHcy levels, tHcy levels significantly increased even among those in the folic acid treatment group over the 4.5-year treatment period. These results suggest that, beyond folic acid therapy, more safe and effective strategies need to be developed for the further control of tHcy levels.

After the adjustment of baseline tHcy, MTHFR C677T did not significantly affect the tHcy-lowering effect of folic acid therapy in our previous study $(n 445)^{(23)}$, nor in the study by Ho et $a l .{ }^{(24)}$ ( $n$ 443). However, the study by Fohr et al. ${ }^{(25)}$ suggested that, although the three genotype groups did not differ significantly in tHcy concentration at baseline, subjects with the TT genotype still responded with a larger decrease in tHcy levels than did those without the mutation. The present study, which included a total of 16644 participants and is by far the largest study of its kind, concluded that even with the adjustment of baseline tHcy, folate and other important covariates, those participants with the TT genotype had a stronger tHcy reduction than those with either the CC or TT genotypes. However, the modifying effect of the MTHFR C677T genotype was weaker and became insignificant in participants with lower baseline tHcy levels. Furthermore, a lower degree of tHcy increase in participants with lower baseline tHcy (Fig. 3) and a greater degree of tHcy reduction in participants with higher baseline tHcy (Fig. 2) was also found in those with lower folate levels, eGFR $<60 \mathrm{ml} / \mathrm{min}$ per $1.73 \mathrm{~m}^{2}$, males and ever smokers, independent of baseline tHcy levels. However, more studies are needed to confirm our results, and to further detect the underlying mechanisms involved in these modifying effects.

In folate-replete populations, the main determinant of elevated tHcy is metabolic vitamin $B_{12}$ deficiency ${ }^{(16,26)}$. This is very common and quite often missed because physicians tend to assume incorrectly that a serum $\mathrm{B}_{12}$ in the reference range represents adequacy of $\mathrm{B}_{12}$ function ${ }^{(27)}$. In the present study, the tHcy-lowering effect of folic acid therapy was very attenuated and became insignificant in those with metabolic $\mathrm{B}_{12}$ deficiency. These results highlight the importance of using vitamin $\mathrm{B}_{12}$ in addition to folic acid to lower tHcy. Moreover, methylcobalamin should be used in place of cyanocobala$\min ^{(28)}$, particularly in persons with impaired renal function (which includes the elderly) ${ }^{(29)}$.

The mean tHcy reduction $(-1.6 \mu \mathrm{mol} / \mathrm{l})$ in the CSPPT is relatively small compared with other published trials; we offer the following explanations. First, the CSPPT supplemented with folic acid alone, whereas most other studies combined folate, $\mathrm{B}_{6}$ and/or $\mathrm{B}_{12}{ }^{(10-12)}$. The addition of other $\mathrm{B}$ vitamins may have helped to further lower the tHcy levels in these studies (about $7 \%$ more than folic acid supplementation alone $)^{(13)}$. Second, there are important differences in characteristics between the participants of the CSPPT and those of previously published trials, including younger age, lower percentage of males and relatively higher eGFR levels ${ }^{(10-12)}$. We have found that these factors can significantly modify the effect of folic acid 


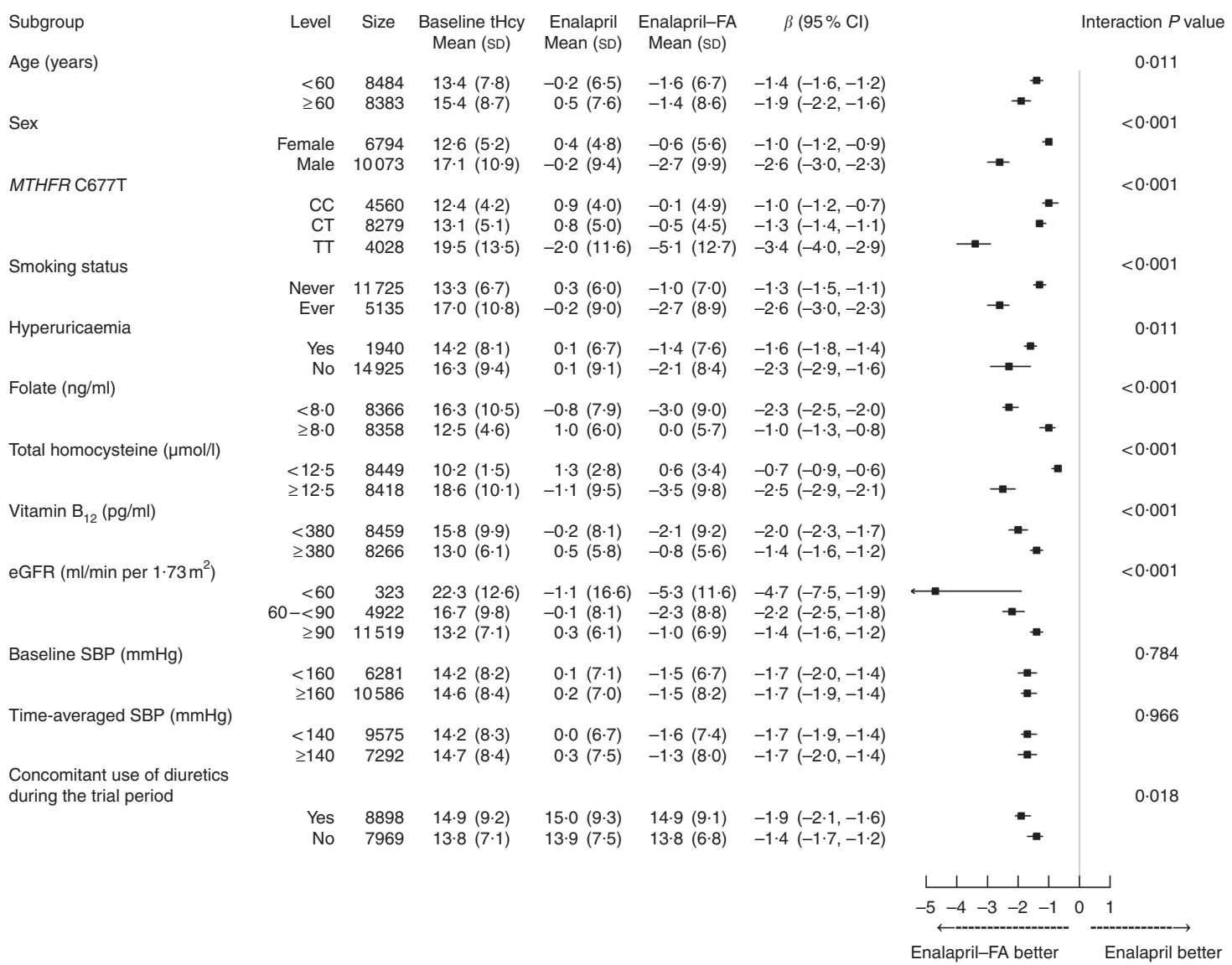

Fig. 1. Stratified analyses by potential effect modifiers. Adjusted, if not stratified, for age, sex, study centre, use of angiotensin-converting enzyme inhibitors, methylenetetrahydrofolate reductase (MTHFR) C677T genotypes, smoking status, BMI, total homocysteine (tHcy), fasting glucose, total cholesterol, estimated glomerular filtration rate (eGFR), folate, vitamin $\mathrm{B}_{12}$, uric acid and systolic blood pressure (SBP) at baseline, as well as time-averaged SBP, concomitant use of calcium channel blockers and concomitant use of diuretics during the trial period. Hyperuricaemia was defined as a uric acid concentration $\geq 417 \mu \mathrm{mol} / \mathrm{l}(7 \mathrm{mg} / \mathrm{dl})$ in $\mathrm{men}$ or $\geq 357 \mu \mathrm{mol} / \mathrm{l}(6 \mathrm{mg} / \mathrm{dl})$ in women. FA, folic acid.

supplementation in lowering tHcy. Last, in the CSPPT, there was a $54 \%$ increase in folate level in the control group during the course of the trial ${ }^{(14)}$. The cause of this increase is unclear. Over the course of the study, subjects received nutritional health education, which may have led to improved dietary choices ${ }^{(29,30)}$. Regardless of the cause, this change diminished the contrast between the two treatment groups and probably attenuated the effect estimation. Therefore, the potential true effect of folic acid supplementation on tHcy reduction could be underestimated in this setting.

The CSPPT found that the combined use of enalapril and folic acid, compared with enalapril alone, significantly reduced the risk of first stroke by $21 \%$ (hazard ratio (HR) 0.79; $95 \%$ CI 0.68, 0.93 ) among hypertensive patients ${ }^{(14)}$. Our previous post hoc analysis of the CSPPT demonstrated that percent decline in tHcy was significantly associated with a reduction in stroke risk in hypertensive patients ${ }^{(12)}$. When percentage decline in tHcy was assessed as tertiles, a significantly lower stroke risk was found in those in tertiles 2 and 3 (HR 0.79; 95\% CI 0.64, 0.97) compared with participants in tertile $1^{(12)}$. Accordingly, tHcy reduction can be considered a reliable biomarker that indicates the beneficial effect of folic acid therapy in the prevention of stroke. However, in the CSPPT, the group difference in tHcy was only $1.6 \mu \mathrm{mol} / \mathrm{l}$ (about an $11 \%$ reduction); therefore, we speculate that thcy reduction may only partially explain the stroke risk reduction associated with folic acid therapy. We cannot ignore the direct antioxidant and antithrombotic effects of folate ${ }^{(31)}$. More studies and analyses are needed to further illuminate the benefits of folate, independent of tHcy lowering, on the risk of stroke.

Our study had several strengths. First, the CSPPT included hypertensive participants without pre-existing stroke or MI. The low vascular disease burden and the low frequency of cardiac and vascular protective drug use made our results less likely to be confounded by these drugs. Second, the CSPPT, which had a large sample size and obtained individual data on MTHFR C677T genotypes, folate, vitamin $\mathrm{B}_{12}$, tHcy levels and other important factors at baseline, offers an exceptional opportunity to comprehensively evaluate potential effect modifiers for the tHcy-lowering effect of folic acid supplementation. Third, the CSPPT measured tHcy levels for $98.7 \%$ of participants at baseline and $81.5 \%$ of participants at the exit visit ${ }^{(14)}$. Most relevant trials, however, only measured tHcy in a fraction of the total participants ${ }^{(32-34)}$. Therefore, the tHcy levels reported by these trials might not represent the whole spectrum of folate and tHcy levels in the total participants. 


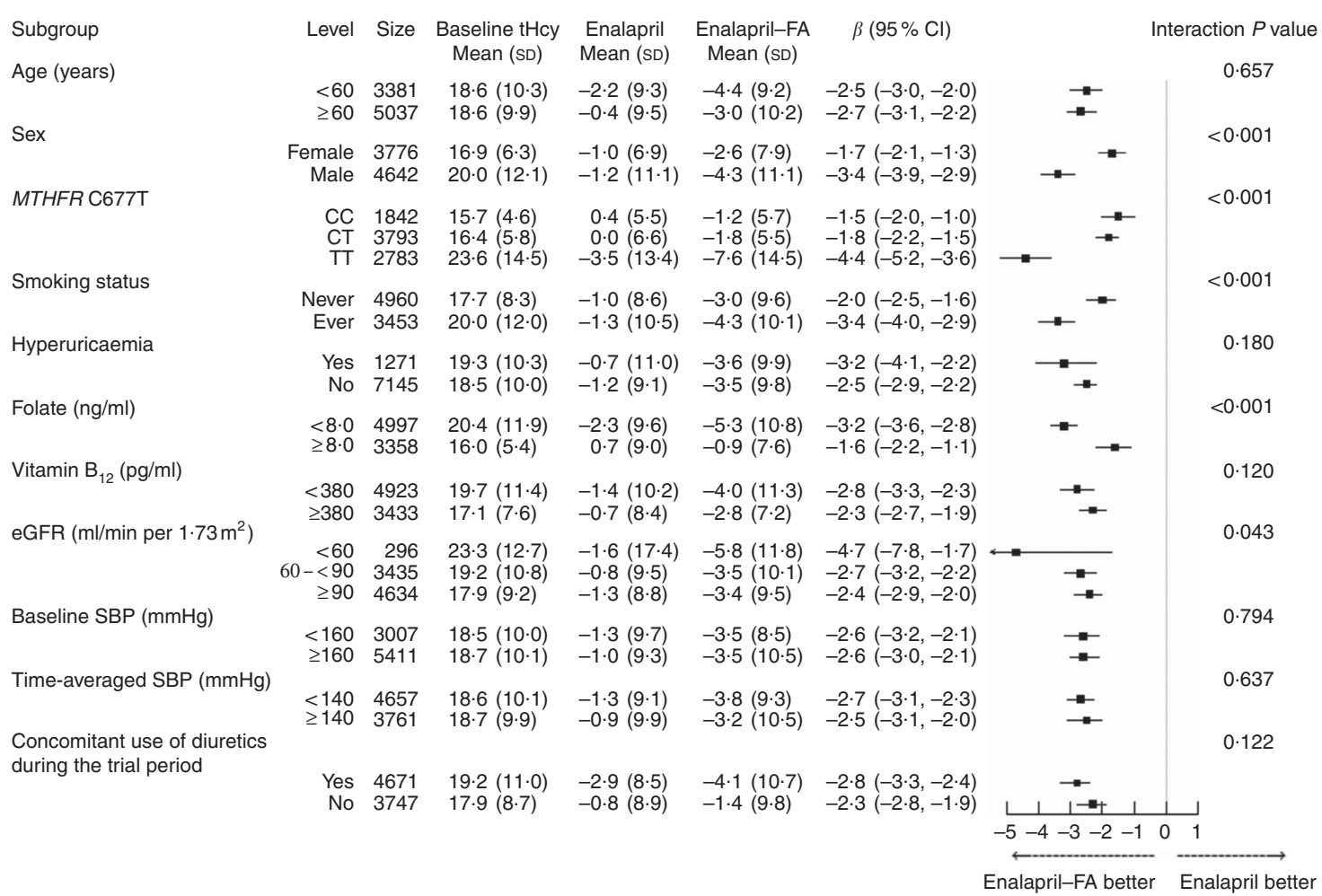

Fig. 2. Stratified analyses by potential effect modifiers in patients with higher baseline total homocysteine (tHcy) levels $(\geq 12.5 \mu$ mol/l). Adjusted, if not stratified, for age, sex, study centre, use of angiotensin-converting enzyme inhibitors, methylenetetrahydrofolate reductase (MTHFR) C677T genotypes, smoking status, BMI, tHcy, fasting glucose, total cholesterol, estimated glomerular filtration rate (eGFR), folate, vitamin $B_{12}$, uric acid and systolic blood pressure (SBP) at baseline, as well as time-averaged SBP, concomitant use of calcium channel blockers and concomitant use of diuretics during the trial period. Hyperuricaemia was defined as a uric acid concentration $\geq 417 \mu \mathrm{mol} / / \mathrm{l}(7 \mathrm{mg} / \mathrm{dl})$ in $\mathrm{men}$ or $\geq 357 \mu \mathrm{mol} / /(6 \mathrm{mg} / \mathrm{dl})$ in women. FA, folic acid.

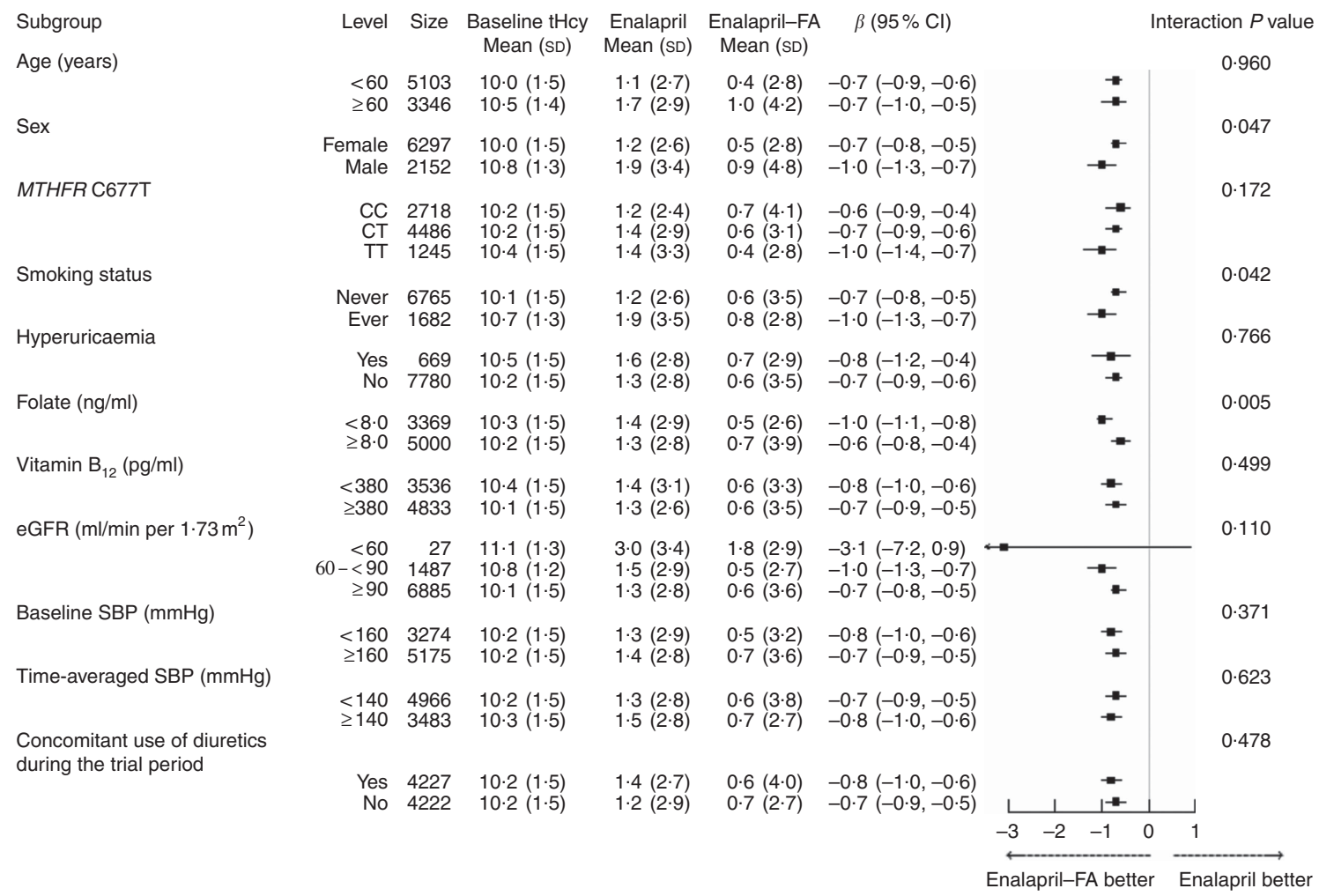

Fig. 3. Stratified analyses by potential effect modifiers in patients with lower baseline total homocysteine (tHcy) levels $(<12 \cdot 5 \mu \mathrm{mol} / /)$. Adjusted, if not stratified, for age, sex, study centre, use of angiotensin-converting enzyme inhibitors, methylenetetrahydrofolate reductase (MTHFR) C677T genotypes, smoking status, BMI, tHcy, fasting glucose, total cholesterol, estimated glomerular filtration rate (eGFR), folate, vitamin $B_{12}$, uric acid and systolic blood pressure (SBP) at baseline, as well as time-averaged SBP, concomitant use of calcium channel blockers and concomitant use of diuretics during the trial period. Hyperuricaemia was defined as a uric acid concentration $\geq 417 \mu \mathrm{mol} / \mathrm{l}(7 \mathrm{mg} / \mathrm{dl})$ in $\mathrm{men}$ or $\geq 357 \mu \mathrm{mol} / \mathrm{l}(6 \mathrm{mg} / \mathrm{dl})$ in women. FA, folic acid. 
The present study also had some limitations. First, this study focused on adults with hypertension; the generalisability of our results to adults without hypertension remains to be determined. However, SBP both at baseline and over the treatment period did not significantly affect the tHcy-lowering effect of folic acid supplementation. Second, we did not measure other polymorphisms in the folate pathway, such as the MTHFR A1298C variant. Overall, our results still need to be investigated and confirmed in future studies.

In conclusion, long-term daily $0.8 \mathrm{mg}$ folic acid supplementation can significantly lower tHcy levels in a population of Chinese hypertensive patients with no history of CVD. More importantly, the degree of tHcy reduction is highly variable, and can be significantly affected by sex, MTHFR C677T genotypes, baseline folate, eGFR and smoking status. All these factors should be taken into account in the identification of participants who may be most likely to benefit from therapy to lower tHcy. The present study, therefore, provides useful data to inform the debate on folic acid fortification for public health reasons and folic acid supplementation for the prevention of a variety of clinical outcomes.

\section{Acknowledgements}

This work was supported by the National Key Research and Development Program (2016YFC0903103, 2016YFC0904900, 2016YFE0205400); the Science and Technology Planning Project of Guangzhou, China (201707020010); and the Science, Technology and Innovation Committee of Shenzhen (KQCX20120816105958775, JSGG20160229173428526, JSGG20170412155639040, GJHS20170314114526143, KC2014JSCX0071A); President Foundation of Nanfang Hospital, Southern Medical University (2017C007); Outstanding Youths Development Scheme of Nanfang Hospital, Southern Medical University (2017J009).

The funders had no role in the design and conduct of the study (data collection, management, analysis and interpretation); the preparation, review or approval of the manuscript; or the decision to submit the manuscript for publication.

X. Q., X. X., B. W., Y. L., X. H., J. L., Y. Z., Y. C., M. H., G. T., D. Y., J. L. and Y. H. contributed to research idea and study design. B. W., Y. L., X. H., H. W., Q. B., L. C., J. L., Y. Z., M. H., G. T., D. Y., Y. H. and X. Q. were involved in data acquisition. B. W., Y. L., X. H., H. W., Q. B., L. C. and X. Q. were involved in data analysis and interpretation. B. W., H. W., Y. L. and X. Q. performed statistical analysis. B. W., H. W., Y. L., X. H., Q. B., L. C., J. L., Y. Z., Y. C., M. H., G. T., D. Y., J. L., Y. H., X. Q. and $\mathrm{X}$. X. carried out review and revision of the article.

$\mathrm{X} . \mathrm{X}$. reports grants from the Projects of the National Natural Science Foundation of China; the Science and Technology Planning Project of Guangzhou, China; and the Science, Technology and Innovation Committee of Shenzhen. B. W. reports grants from the National Key Research and Development Program. Y. H. reports grants from the National Key Research and Development Program. Y. C. reports grants from National Key Technologies Research and Development Program. X. Q. reports grants from Nanfang Hospital, Southern Medical University. Y. L. reports grants from Nanfang Hospital, Southern Medical University. No other disclosures were reported.

\section{Supplementary material}

For supplementary material/s referred to in this article, please visit https://doi.org/10.1017/S0007114518002477

\section{References}

1. Wald DS, Law M \& Morris JK (2002) Homocysteine and cardiovascular disease: evidence on causality from a metaanalysis. BMJ 325, 1202.

2. Stanger O, Herrmann W, Pietrzik K, et al. (2003) DACH-LIGA homocystein (German, Austrian and Swiss Homocysteine Society): consensus paper on the rational clinical use of homocysteine, folic acid and B-vitamins in cardiovascular and thrombotic diseases: guidelines and recommendations. Clin Chem Lab Med 41, 1392-1403.

3. Xie D, Yuan Y, Guo J, et al. (2015) Hyperhomocysteinemia predicts renal function decline: a prospective study in hypertensive adults. Sci Rep 5, 16268

4. Towfighi A, Markovic D \& Ovbiagele B (2010) Pronounced association of elevated serum homocysteine with stroke in subgroups of individuals: a nationwide study. J Neurol Sci 298, 153-157.

5. Li J, Jiang S, Zhang Y, et al. (2015) H-type hypertension and risk of stroke in Chinese adults: a prospective, nested casecontrol study. J Transl Int Med 3, 171-178.

6. Qin X, Li Y, Sun N, et al. (2017) Elevated homocysteine concentrations decrease the antihypertensive effect of angiotensin-converting enzyme inhibitors in hypertensive patients. Arterioscler Thromb Vasc Biol 37, 166-172.

7. Li Y, Huang T, Zheng Y, et al. (2016) Folic acid supplementation and the risk of cardiovascular diseases: a meta-analysis of randomized controlled trials. J Am Heart Assoc 5, e003768.

8. Qin X, Xu M, Zhang Y, et al. (2012) Effect of folic acid supplementation on the progression of carotid intima-media thickness: a meta-analysis of randomized controlled trials. Atherosclerosis 222, 307-313.

9. Huang X, Li Y, Li P, et al. (2017) Association between percent decline in serum total homocysteine and risk of first stroke. Neurology 89, 2101-2107.

10. Huo Y, Qin X, Wang J, et al. (2012) Efficacy of folic acid supplementation in stroke prevention: new insight from a meta-analysis. Int J Clin Pract 66, 544-551.

11. Yang HT, Lee M, Hong KS, et al. (2012) Efficacy of folic acid supplementation in cardiovascular disease prevention: an updated meta-analysis of randomized controlled trials. Eur J Intern Med 23, 745-754

12. Zhao M, Wu G, Li Y, et al. (2017) Meta-analysis of folic acid efficacy trials in stroke prevention: insight into effect modifiers. Neurology 88, 1830-1838.

13. Homocysteine Lowering Trialists' Collaboration (2005) Dosedependent effects of folic acid on blood concentrations of homocysteine: a meta-analysis of the randomized trials. $\mathrm{Am} \mathrm{J}$ Clin Nutr 82, 806-812.

14. Huo Y, Li J, Qin X, et al. (2015) Efficacy of folic acid therapy in primary prevention of stroke among adults with hypertension in China: the CSPPT randomized clinical trial. JAMA $\mathbf{3 1 3}$, $1325-1335$

15. Xu X, Qin X, Li Y, et al. (2016) Efficacy of folic acid therapy on the progression of chronic kidney disease: the renal substudy of the China Stroke Primary Prevention Trial. JAMA Intern Med 176, 1443-1450.

16. Robertson J, Iemolo F, Stabler SP, et al. (2005) Vitamin $\mathrm{B}_{12}$, homocysteine and carotid plaque in the era of folic acid fortification of enriched cereal grain products. CMAJ 172, 1569-1573. 
17. Refsum H, Smith AD, Ueland PM, et al. (2004) Facts and recommendations about total homocysteine determinations: an expert opinion. Clin Chem 50, 3-32.

18. Mathews F, Yudkin P, Smith RF, et al. (2000) Nutrient intakes during pregnancy: the influence of smoking status and age. J Epidemiol Community Health 54, 17-23.

19. Ortega RM, Requejo AM, Lopez-Sobaler AM, et al. (2004) Smoking and passive smoking as conditioners of folate status in young women. J Am Coll Nutr 23, 365-371.

20. Yanbaeva DG, Dentener MA, Creutzberg EC, et al. (2007) Systemic effects of smoking. Chest 131, 1557-1566.

21. Ulvik A, Ebbing M, Hustad S, et al. (2010) Long- and shortterm effects of tobacco smoking on circulating concentrations of B vitamins. Clin Chem 56, 755-763.

22. Homocysteine Lowering Trialists' Collaboration (1998) Lowering blood homocysteine with folic acid based supplements: meta-analysis of randomised trials. BMJ 316, 894-898.

23. Qin X, Li J, Cui Y, et al. (2012) MTHFR C677T and MTR A2756G polymorphisms and the homocysteine lowering efficacy of different doses of folic acid in hypertensive Chinese adults. Nutr J 11, 2.

24. Ho GY, Eikelboom JW, Hankey GJ, et al. (2006) Methylenetetrahydrofolate reductase polymorphisms and homocysteinelowering effect of vitamin therapy in Singaporean stroke patients. Stroke 37, 456-460.

25. Fohr IP, Prinz-Langenohl R, Bronstrup A, et al. (2002) 5,10Methylenetetrahydrofolate reductase genotype determines the plasma homocysteine-lowering effect of supplementation with 5-methyltetrahydrofolate or folic acid in healthy young women. Am J Clin Nutr 75, 275-282.
26. Quinlivan EP, McPartlin J, McNulty H, et al. (2002) Importance of both folic acid and vitamin $B_{12}$ in reduction of risk of vascular disease. Lancet 359, 227-228.

27. Spence JD, Yi Q \& Hankey GJ (2017) B vitamins in stroke prevention: time to reconsider. Lancet Neurol 16, 750-760.

28. Spence JD, Urquhart BL \& Bang H (2016) Effect of renal impairment on atherosclerosis: only partially mediated by homocysteine. Nephrol Dial Transplant 31, 937-944.

29. Qin X, Zhang Y, Cai Y, et al. (2013) Prevalence of obesity, abdominal obesity and associated factors in hypertensive adults aged 45-75 years. Clin Nutr 32, 361-367.

30. Qin X, Li J, Spence JD, et al. (2016) Folic acid therapy reduces the first stroke risk associated with hypercholesterolemia among hypertensive patients. Stroke 47, 2805-2812.

31. Verhaar MC, Stroes E \& Rabelink TJ (2002) Folates and cardiovascular disease. Arterioscler Thromb Vasc Biol 22, 6-13.

32. Albert CM, Cook NR, Gaziano JM, et al. (2008) Effect of folic acid and B vitamins on risk of cardiovascular events and total mortality among women at high risk for cardiovascular disease: a randomized trial. JAMA 299, 2027-2036.

33. Study of the Effectiveness of Additional Reductions in Cholesterol and Homocysteine (SEARCH) Collaborative Group, Armitage JM, Bowman L, et al. (2010) Effects of homocysteinelowering with folic acid plus vitamin $\mathrm{B}_{12}$ vs placebo on mortality and major morbidity in myocardial infarction survivors: a randomized trial. JAMA 303, 2486-2494.

34. Bostom AG, Carpenter MA, Kusek JW, et al. (2011) Homocysteine-lowering and cardiovascular disease outcomes in kidney transplant recipients: primary results from the Folic Acid for Vascular Outcome Reduction in Transplantation trial. Circulation 123, 1763-1770. 